\title{
Conflictos socioambientales en las microcuencas de Villavicencio 1980-2015
}

\section{Socio-environmental conflicts in the micro-basins of Villavicencio 1980-2015}

\author{
Juan Manuel Ochoa Amaya ${ }^{1}$ \\ Rubén Darío Cely Velásquez ${ }^{2}$ \\ Luz Gladys Yarime Peña Ulloa ${ }^{3}$
}

\begin{abstract}
$1 \quad$ Doctor $(\odot)$ Estudios Territoriales (Universidad de Caldas); MSc Desarrollo Sostenible y Medio Ambiente (Universidad de Manizales); Economista (Universidad Pedagógica y Tecnológica de Colombia); Profesor de planta Universidad de los Llanos (Colombia). Hace parte del Grupo de Investigación "CERES" (Universidad de Caldas); Líder Grupo de Investigación Territorio y Ambiente (Universidad de los Llanos). Correo electrónico: juan.ochoa@unillanos. edu.co. ORCID ID: https://orcid.org/0000-0001-7198-0577. Google Scholar: https://scholar.google.es/citations?user=atmB3W8AAAAJ\&hl=es\&oi=ao
\end{abstract}

$2 \quad$ Magíster en Estudios de Desarrollo Local (Universidad de los Llanos); Ingeniero Ambiental (Universidad del Meta). Hace parte del Grupo de Investigación Territorio y Ambiente (Universidad de los Llanos). Correo electrónico: ruben.cely@unillanos.edu.co. ORCID ID: https://orcid. org/0000-0002-3496-0638.

Google Scholar: https://scholar.google.es/citations?view_ op=list_works\&hl=es\&user=jmfAP1AAAAAJ

3 Magíster en Economía (Universidad de Manizales); Economista; Profesora Universidad de los Llanos (Colombia). Hace parte del Grupo de Investigación Territorio y Ambiente (Universidad de los Llanos), Correo electrónico: yarime.pena@unillanos.edu.co https://orcid.org/0000-00033628-6277. Google Scholar: https://scholar.google.es/citations?view_op=list_works\&hl=es\&user=7cW03C0AAAAJ

\section{RESUMEN}

El presente artículo identifica distintas acciones de los actores sociales como prácticas socioeconómicas, culturales y procesos de apropiación de los recursos naturales en la microcuenca del Zuria en Villavicencio; pudiéndose determinar que los procesos de territorialización se relacionan dentro de una triada geo-socio-histórica.

El sustento teórico se cimienta sobre las bases del modelo económico desarrollista, causante de distintos problemas ambientales sobre la configuración en los distintos ecosistemas y sus recursos naturales, alterando la biodiversidad natural al ser reemplazada por otro tipo de plantas o cultivos de explotación económica, 
que afectaron la estabilidad de los suelos, especialmente por el uso de plaguicidas, lo cual a la postre significó terrenos infértiles y cambio en la vocación y uso del suelo.

El trabajo presentado se basó en el estudio de mapas, estructurado en las siguientes fases: identificación de la unidad de análisis representada en la delimitación del corredor biológico Zuria; consolidación de información geográfica multitemporal a través de la aplicación metodológica Corine Land Cover para la clasificación de coberturas vegetales; análisis de información proyectada por medio de salidas gráficas para los periodos 1992, 2002, 2007 y 2012, basadas en resultados previos consistentes en la descripción, individualización, clasificación y comparación de sus características.

\section{PALABRAS CLAVE}

Microcuenca, territorialización, conflicto socioambiental, desarrollismo.

\section{ABSTRACT}

This article identifies different actions of social actors such as socio-economic and cultural practices and processes of appropriation of natural resources in the micro-basin of Zuria in Villavicencio; being able to determine that the territorialization processes are related within a geo-socio-historical triad.

The theoretical support is based on the bases of the developmental economic model, causing different environmental problems on the configuration of the different ecosystems and their natural resources, altering the natural biodiversity by being replaced by other types of plants or crops of economic exploitation, which affected the stability of the soils, especially due to the use of pesticides, which ultimately meant infertile lands and a change in the vocation and use of the soil.
The work presented was based on the study of maps, structured in the following phases: identification of the unit of analysis represented in the delimitation of the Zuria biological corridor; consolidation of multitemporal geographic information through the Corine Land Cover methodological application for the classification of vegetal covers; analysis of projected information through graphic outputs for the periods 1992, 2002, 2007 and 2012, based on previous results consisting of the description, individualization, classification and comparison of its characteristics.

Keywords: Micro-basin, territorialization, socioenvironmental conflict, developmentalism.

\section{INTRODUCCIÓN}

La microcuenca del caño Zuria se encuentra en el municipio de Villavicencio, es tributaria del rio Negro, que a su vez es afluente del rio Ocoa. Como fuente hídrica provee servicios ecosistémicos en toda el área de estudio (buffer), en un espacio de 1370 hectáreas aproximadamente, dividido y trazado de manera heterogénea teniendo en cuenta diferentes fragmentos de coberturas sobre su superficie como enormes capas de cemento, asfalto, pastos, bosques, cultivos y agua, siendo de resaltar este último recurso como uno de los baluartes territoriales de Zuria, principalmente porque gracias a él se desarrollaron las dinámicas territoriales abordadas en la investigación.

De esta forma y durante un periodo de más de cuarenta años actores de diferentes regiones geográficas poblaron el piedemonte de la cordillera oriental, especialmente Villavicencio originando grandes transformaciones en la estructura de sus suelos. Sus costumbres y formas de trabajar la tierra fueron trascendentales en el proceso de reconfiguración territorial, especialmente a través de sus modos de producción. De una parte, desplazados por causa de la crisis política y económica que 
atravesaba el país para la década de los años 50 y posteriores, especialmente campesinos desposeídos en busca de un territorio para construir su hogar y producir. Otras, fueron las víctimas del conflicto armado y social dejado por el narcotráfico de los años 80 , que vieron en Villavicencio un sitio promisorio donde poder comprar un pedazo de tierra para establecerse, vivir y/o invertir.

Esos procesos migratorios permitieron distintas formas de territorialización en Zuria, manifestadas a través de los años en acciones contrarias a las de preservación del medio ambiente, así la tala de árboles, quema de rastrojos, ganadería, siembra de arroz y soya, entre otros reconfiguraron el territorio. Sistemas de penetración territorial sobre la naturaleza, con repercusiones que hoy en día son palpables a través de la fragmentación de fincas y fenómenos de potrerización, las cuales son evidenciadas como conflictos ambientales en el área de estudio.

Conflictos ambientales entendidos como las complicaciones que se generan en un territorio por el impulso de impactos que ocasionan el deterioro sobre los recursos naturales como el agua y el suelo, así como en diversidad de especies animales o vegetales (Universidad de Cantabria, 2017). En Zuria esa distribución de tierras y recursos naturales dio paso a la ampliación de la frontera agrícola, impactando el suelo con los sistemas de mecanización para cultivos, los cuales fueron acompañados con el uso de pesticidas y herbicidas, que finalmente afectaron el agua perjudicando y diezmando la vida acuática.

En este trabajo se logran evidenciar esos fenómenos de afectación antrópica gracias al uso de los sistemas de información geográficos, que implementados como herramienta de trabajo permitieron observar desde diferentes perspectivas, territoriales, ambientales $y$ geográficas el uso del suelo en el lugar de trabajo, el Zuria en este caso como microcuenca hidrográfica, pudiéndose entrelazar superficies heterogéneas con la finalidad de hallar la sinergia territorio - actor en el proceso de reconfiguración espacial.

En esta indagación se tomaron superficies presentadas en Bases de Datos como las del SINCHI para los años 1992, 2002, 2007 y 2012 en Villavicencio. Mostrando claramente cómo ha sido la reconfiguración de la microcuenca del caño Zuria, siendo la base de información que nutre a esta investigación.

El uso de la información cartográfica brindada por el SINCHI sirvió como técnica para revisar escenarios de cambio cronológico, a partir de una adecuada comprensión de los factores económicos, sociales, ambientales e incluso políticos, permitiendo identificar distintos procesos de reconfiguración en Zuria durante el periodo de estudio. Se realizó una clasificación supervisada de coberturas vegetales en la multitemporalidad, que determinó los valores en porcentaje de cada tipo de cobertura, realizando la justificación del patrón de distribución de las coberturas vegetales con respecto al grado de interacción entre las mismas.

Finalmente, el resultado de la superposición permitió observar el cambio de dispersión territorial y la pérdida de coberturas vegetales respecto a la expansión del tejido urbano y las actividades agrícolas en la transición de tiempo establecido, generando como resultado: el análisis de los conflictos socioambientales en la microcuenca del caño Zuria durante los años 1980 al 2015.

\section{EL DEBATE TEÓRICO. ENTRE TERRITORIO, MODELO ECONÓMICO Y RECONFIGURACIÓN TERRITORIAL}

La base teórica que acompaña la presente investigación se orienta al análisis e identificación de las acciones sociales de los actores y sus prácticas socioeconómicas y culturales por 
efectos de apropiación de los recursos naturales en el corredor biológico Zuria. Esgrimiéndose que los procesos de territorialización (19802015) tienen una marcada presencia del modelo de desarrollo enfocado al crecimiento agrícola con generación de excedentes, no siendo más que la presencia del desarrollismo como paradigma del modelo de Desarrollo Económico inhibiendo comportamientos territoriales en esta parte del piedemonte.

Igualmente, la relación territorio y ambiente es una conjunción directa. El territorio es un espacio que contiene procesos de organización social en los cuales el argumento ambiental está presente para la formación de identidad en ese espacio territorial (Flórez, 2007). Con esto se deja entrever que junto con los sistemas de producción agrícolas implementados en el piedemonte de Villavicencio se desarrollaron distintas acciones sociales de los actores en un proceso de apropiación del espacio, es decir, "parece perfectamente sentado que el espacio físico no posee ninguna realidad sin la energía que se despliega dentro de él" (Lefebvre, 2013, p. 74), así el espacio se convierte en espacio social y agrícola gracias al interés puesto por alguien, en este caso una acción proveniente de un proceso de producción campesina.

En concordancia con lo anterior,

Será necesario evidenciar más adelante que este espacio social no consiste en una colección de cosas, en una suma de datos (sensibles), ni tampoco en un vacío colmado (algo así como un envase) de materias diversas, habrá que mostrar que no se reduce a una forma impuesta a los fenómenos, a las cosas, a la materialidad física (Lefebvre, 2013, p. 87).

De forma más precisa, con el desarrollo del capitalismo después de la postguerra fue posible observar procesos de imbricación que dieron lugar a distintas reproducciones sociales generadas por la implementación de un modelo de producción económica, el cual ha sido el precursor de distintos conflictos, enfrentamientos, luchas y guerras (Lefebvre, 2013), principalmente con la implementación de la revolución verde y las sucesivas acciones recaídas sobre los espacios boscosos con la finalidad de ampliar las fronteras de producción agrícola.

Así fue que la revolución verde estuvo presente territorialmente entre 1960 y 1980, extendida para aumentar la producción por unidad de superficie y de trabajo, pero apoyada de instrumentos tecnológicos adaptados a las necesidades de la población rural, quien se encontraba expuesta a la inseguridad alimentaria, permitiéndole a los agricultores de tierras fértiles aprovechar su unidad de trabajo, logrando una mayor productividad agrícola, sostenible económicamente (FAO, 1996).

Con lo anterior, el uso de una serie de prácticas como la producción extensiva a gran escala, cultivos más resistentes a las plagas, al cambio climático, uso de pesticidas y crecimiento del área cultivada a través de los monocultivos. Con ello la producción creció exponencialmente, demandando ahora terrenos como las rondas de los cuerpos hídricos y zonas sin una vocación para la agricultura, pero que con la técnica apropiada cambiaría las características del suelo. Todo ello condujo a muchos territorios a sufrir daños ambientales como deforestación, compactación del suelo, sedimentación del terreno y tierras áridas (Ceccon E. , 2018).

De otra parte, este modelo de producción soportado en teorías desarrollistas originó una serie de desventajas como el impacto medioambiental, principalmente por el uso intensivo de fertilizantes y pesticidas. Igualmente, el sistema de producción demandó grandes cantidades de agua con su posterior contaminación por el vertido de químicos (Montano, 2005), así como salinización y 
destrucción del suelo, deforestación y pérdida de biodiversidad.

Ahora bien, desde los años setenta hasta finales de los ochenta las misiones económicas estadounidenses se incorporaron a los sistemas de producción agrícola, con políticas como el uso de fertilizantes, plaguicidas y semillas híbridas (centro virtual de noticias, 2019), transformándolos a una mayor productividad por uso del suelo. Con todo ello, "los modelos de producción agraria, el régimen de tenencia de tierras, los flujos migratorios campo-ciudad y los efectos para la seguridad alimentaria en el país tienen raíces en las ideas de desarrollo promovidas por las misiones norteamericanas" (Arango Marín, 2005, párrafo 4), situación que no es ajena a los programas productivos en el Zuria, debido a que se tiene en el sector un claro direccionamiento hacia la tecnificación agrícola del territorio y el uso de químicos para el control de enfermedades y plagas en los cultivos.

Los beneficios que aportó este sistema de producción desarrollista fueron evidentes. De una parte, el incremento de la cantidad de cosecha por hectárea sembrada, como también, desde otra arista, el daño al medio ambiente, especialmente con el incremento de energía necesaria para desarrollar este tipo de agricultura, como el requerido para mover la maquinaria agrícola que precisa de combustible fósil, o el necesario para la fabricación de fertilizantes y pesticidas, entre otros (Benavides, 2017).

La biodiversidad natural ha desaparecido, reemplazada por un pequeño número de plantas cultivadas, situación que ha conllevado a múltiples problemas ambientales como la perdida de estabilidad en el suelo y terrenos infértiles, además del cambio de uso del terreno y factores sociales como el desplazamiento en masa, invasiones de terrenos que no son viables para habitar, aumento en la mendicidad, entre otras problemáticas.
De otra parte, el crecimiento de Villavicencio se ha venido incrementando de una manera exponencial, especialmente por el desplazamiento forzado y la búsqueda de nuevas oportunidades, además de la expansión de cultivos en zonas rurales generando demanda de terrenos y formando asentamientos ilegales que invaden las rondas de los caños, todo esto acompañado de la falta de inspección territorial y control ambiental, en lo que anteriormente era terreno de bosque y ronda de los caños para desarrollar ahora diferentes actividades económicas (Ochoa, 2014).

Así mismo,

el manejo de los recursos naturales por parte de las sociedades organizadas ha sido una práctica inherente al devenir de la humanidad contra la creencia común, el aprovechamiento milenario de estos recursos ha permitido su conservación a la vez que el desarrollo de importantes culturas (Álvarez-Icaza, 2014, p. 199);

Lo expuesto se puede refutar hoy en día, especialmente porque un crecimiento económico acelerado compromete los recursos naturales y la explotación es superior a la tasa de resiliencia del territorio.

Con referencia a lo mencionado, el ecosistema de la microcuenca del caño Zuria establece una heterogeneidad, otorgando una propiedad de diversificación de la estructura del paisaje basado en un gradiente diferencial o en separaciones ecotonales abruptas y discretas (Bennett, 1999); estos sistemas abiertos heterogéneos se fundamentan en insumos pasados y presentes de energía solar, generalmente modificados por la acción humana en el ambiente (Benayas del Alamo, 1992). La modificación hecha sobre la matriz original del piedemonte se diversifica 
en forma de gradiente (o serie de gradientes) o como un mosaico en el que cada unidad se diferencia por los discretos límites que las identifican (Universidad Nacional de Colombia, 2016).

La visión e interpretación desarrollada desde la ecología asociado con los actores sociales se fundamenta en una aproximación de carácter estructural morfológica, funcional, dinámico, y de manejo los cuales se clasifican por diferentes valores (Subirós y Linde, 2006). Los valores estructurales traen consigo diferentes características como riqueza el cual es el número de clases de elementos ecológicos en una unidad territorial de referencia; en donde la diversidad es la medida de la variedad de elementos que se presentan en el lugar y la uniformidad de su distribución, referida a una reconfiguración del territorio y el teselado con el cual obtiene una conformación espacial de elementos respecto a los cambios que se produjeron anteriormente.

Los valores dinámicos presentan una resiliencia para retornar al estado anterior antes de ser cambiados, esto es el resultado de tendencias generales en el tiempo con fluctuaciones más o menos amplias y una fragilidad de pérdida de biodiversidad cuando el territorio sufre una perturbación (la biodiversidad es sensible a cambios estructurales). Como último valor se presentan los valores de manejo: los cuales se centran en la conservación, productividad y sostenibilidad (Bennett, 1999).

En cuanto a la demanda por tierras cerca de Villavicencio, ha venido aumentando debido principalmente al auge de la construcción, lo que ha presionado el uso del suelo, de manera que el fenómeno de utilización de las tierras traspasa las barreras ambientales ya que, por la necesidad de espacios para la construcción de viviendas de tipo rural, y sobre todo para proyectos urbanísticos, se está presionando también el margen de los ríos, humedales y quebradas. Por su parte Lefebvre (2013) afirma que "la ciudad y el espacio urbano son propios de un dominio especializado: el urbanismo. En cuanto al espacio más amplio, el territorio (regional, nacional, continental o mundial) es competencia de los planificadores y los economistas" (p. 73), lo que reduce drásticamente el hábitat de diferentes especies en áreas rurales y urbanas, además de que presiona los corredores biológicos hasta en algunos casos llegar a su desertización, sin contar la sedimentación de los lechos cuyos efectos devastadores se sintieron con especial fuerza en las inundaciones que sufrió Colombia a finales de 2010, aunque la región de estudio estuvo menos golpeada que el resto del País.

\section{RESULTADOS. EL ANÁLISIS DE LOS CONFLICTOS SOCIOAMBIENTALES EN LA MICROCUENCA DEL CAÑO ZURIA}

Para la comprensión del fenómeno socioespacial estudiado, se realizó un análisis al territorio partiendo sobre la base procedimental Corine Land Cover (CLC). Ésta define una metodología específica que permite plasmar el inventario de cobertura de la tierra, así como la posibilidad de describir, determinar, clasificar y comparar sus características, las cuales se interpretan "a partir de la utilización de imágenes de satélite de resolución media (Landsat), para la construcción de mapas de cobertura a diferentes escalas" (IDEAM, IGAC, CORMAGDALENA, 2007, P. 29).

\subsection{Comparativos históricos sobre las transformaciones de la cobertura vegetal en la cuenca del caño Zuria}

Antes de iniciar la lectura de las imágenes del Zuria, es importante dejar en contexto que todas tienen un encierro o sector delimitado con una línea de color rojo, la cual enmarca la totalidad del área de la microcuenca del caño. El área sobre el cual se desarrolló el trabajo geográfico 
comprende el total de la microcuenca, mientras que los 300 metros a cada uno de sus costados permitió el desarrollo del tratamiento del trabajo presencial sobre el territorio; igualmente, al haberse trabajado con la metodología del Instituto Amazónico de Investigaciones Científicas SINCHI, fue necesario hacer este tipo de demarcación.
Para la comprensión del fenómeno que se presenta, las transformaciones en la cobertura vegetal en Zuria exponen y comparan los mapas de cobertura vegetales en los años 1992, 2002, 2007 y 2012 como se muestra a continuación los cuales fueron procesados a través del software ArcGIS versión 10.4 .

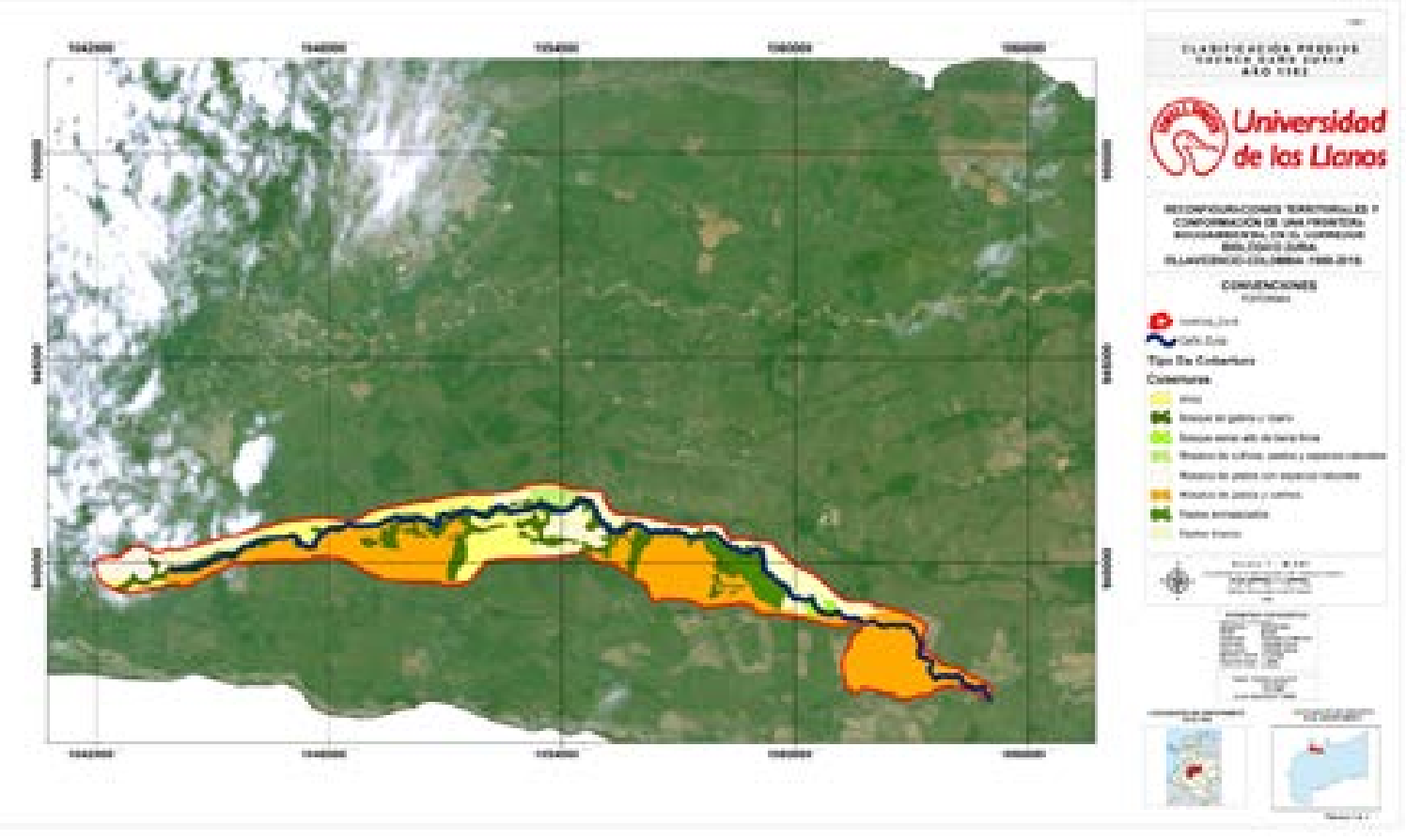

Figura 1. Distribución de coberturas en el área de estudio a 1992. Adaptado de "Estudio de Coberturas Multitemporales 1992-2012", Geodatabase- 2019 por el Instituto Amazónico de Investigaciones Científicas. Fuente: Elaboración Propia. Grupo de Investigación Territorio y Ambiente

Según lo evidenciado en la figura 1, para el año 1992 en alrededores del caño Zuria predominaban con potencia los mosaicos de pastos, cultivos y espacios naturales en un $48.77 \%$, que sumado a los pastos (limpios o enmalezados) superaban el $66 \%$, comprobando los grandes procesos de transformación antrópicos que empezaban a darse sobre estos suelos por la transición agrícola que estaba viviendo el país para ese entonces. Escenario que reconoce en gran parte la vocación del territorio para la producción de alimentos agrícolas y de ganadería vacuna, especialmente una agricultura abundante con un crecimiento exponencial, empezando a ocupar terrenos como las rondas de los cuerpos hídricos y zonas de vocación no agrícola, iniciando un cambio en las características del suelo con posteriores daños ambientales como deforestación, compactación del suelo y sedimentación del terreno (Ceccon E., 2018). 

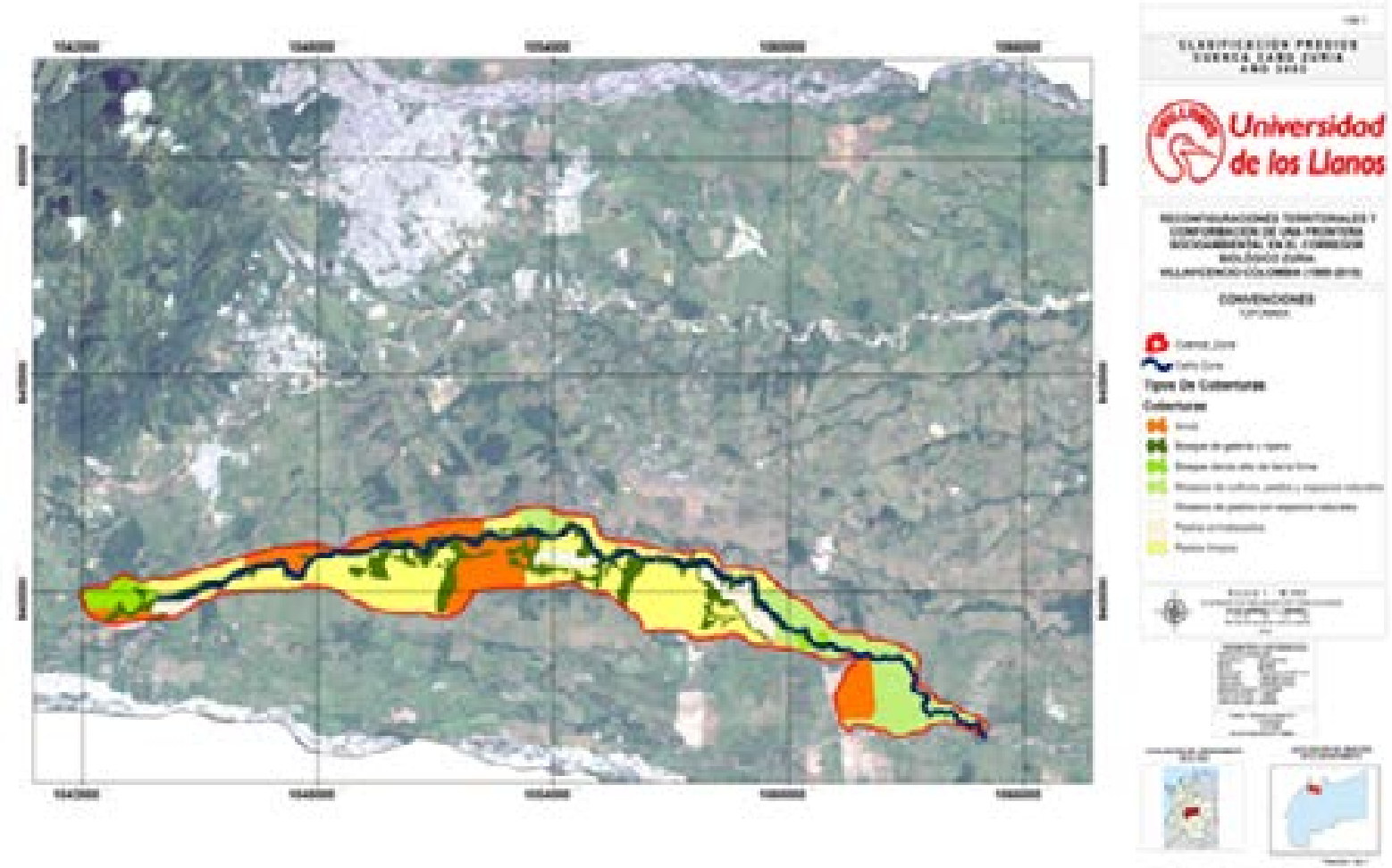

Figura 2. Distribución de coberturas en el área de estudio a 2002. Adaptado de "Estudio de Coberturas Multitemporales 1992-2012", Geodatabase- 2019 por el Instituto Amazónico de Investigaciones Científicas. Fuente: Elaboración Propia. Grupo de Investigación Territorio y Ambiente

En el año 2002, de acuerdo con lo evidenciado en la figura 2 se observan vastas desproporciones en la cobertura vegetal a causa de la expansión del cultivo de arroz, con ocasión a las políticas económicas extendidas territorialmente a través de la reforma agraria de 1994, donde se subsidiaron adquisiciones de tierras para la siembra del cereal. De allí el aumento en $112 \mathrm{Ha}$ aproximadamente, logrando alcanzar el $16 \%$ del total de la microcuenca, expansión que se soportó con procesos de facturación acelerada del grano a nivel nacional. De acuerdo con (Tubio, 2001) la exportación de nuevos productos diferentes a los tradicionales (de pancoger como maíz, yuca, plátano) creó la posición de lugares específicos de dominancia antrópica sobre la naturaleza. Nada ajeno a los programas productivos en el sector del Zuria, fundamentado en que se tiene en el sector un claro direccionamiento hacia la tecnificación agrícola del territorio y el uso de químicos para el control de enfermedades y plagas en los cultivos. 


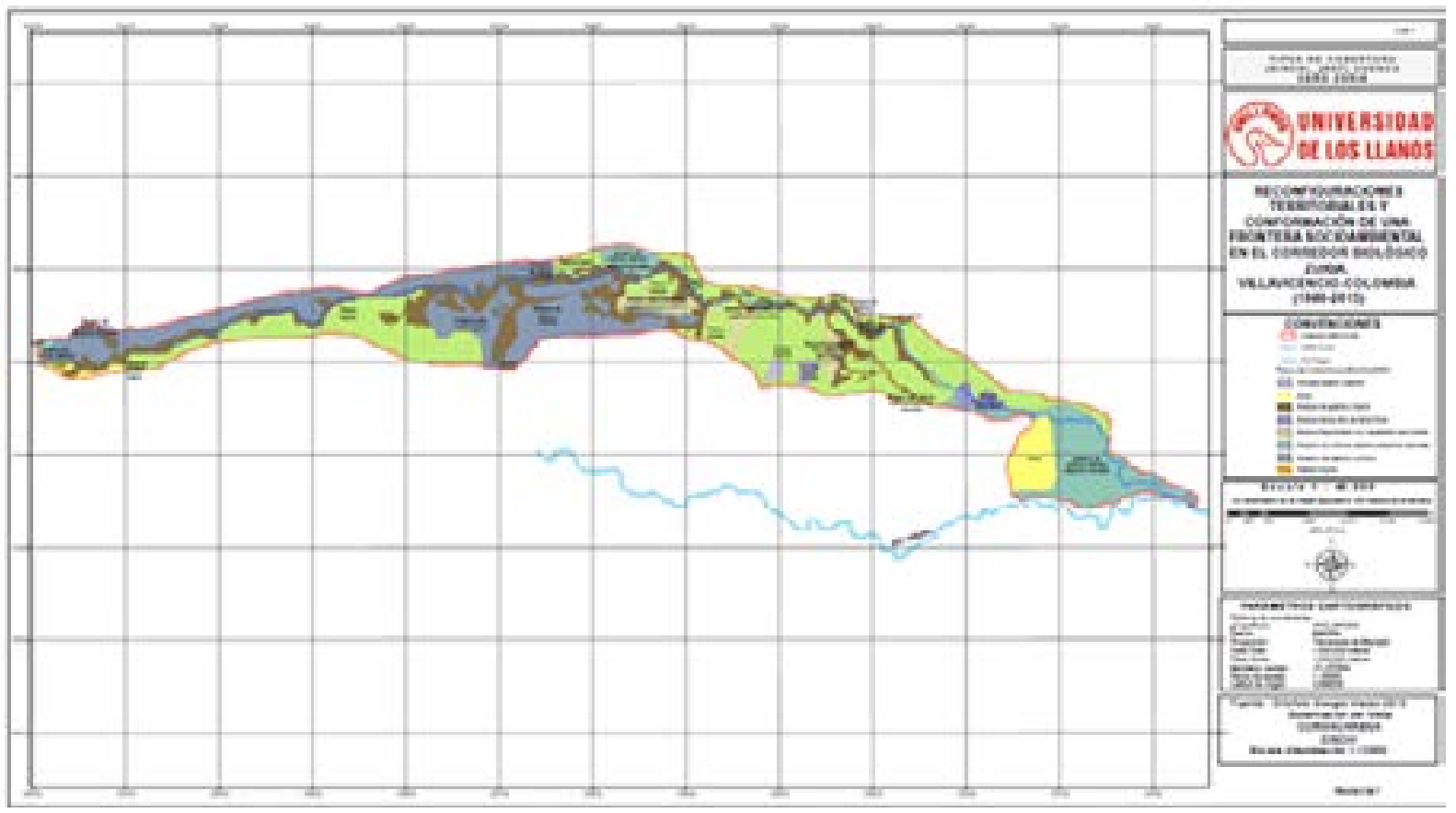

Figura 3. Distribución de coberturas en el área de estudio a 2007. Adaptado de "Estudio de Coberturas Multitemporales 1992-2012", Geodatabase- 2019 por el Instituto Amazónico de Investigaciones Científicas. Fuente: Elaboración Propia. Grupo de Investigación Territorio y Ambiente

El naciente tejido urbano en la década del noventa empieza a desarrollarse con fuerza para el año 2007 en el sector, siendo el corredor ecológico quien sufre la repercusión socioeconómica al depender de la producción ganadera. Una transición fuerte que se presentó fue la de mosaico de cultivos, pastos y espacios naturales al pasar del $21 \%$ en 2002 , al $34,9 \%$ en 2007 como se observa en la figura 3, reduciendo a su vez el cultivo de arroz del $16 \%$ a $6 \%$ respectivamente y los bosques en una unidad porcentual cada uno, evidenciando la introducción de la vocación ganadera al territorio que generó detrimentos en el bosque de galería y ripario, lo cual terminó por impactar el agua, el suelo y la fauna nativa existente en Zuria.

De otra parte, los procesos de expansión territorial han estado acompañados de desplazamiento poblacional, migración y riesgo ambiental en esas zonas que no son aptas para la urbanización, escenario que ha propiciado cambios significativos en el territorio (Restrepo,
2014), lo cual se traduce para las últimas décadas en un crecimiento desordenado y acelerado de las zonas urbanas, impactando de manera significativa la estructura de los territorios ubicados a lo largo de su periferia con múltiples problemáticas como el desplazamiento de actividades económicas en sectores como humedales y rondas de los ríos. 

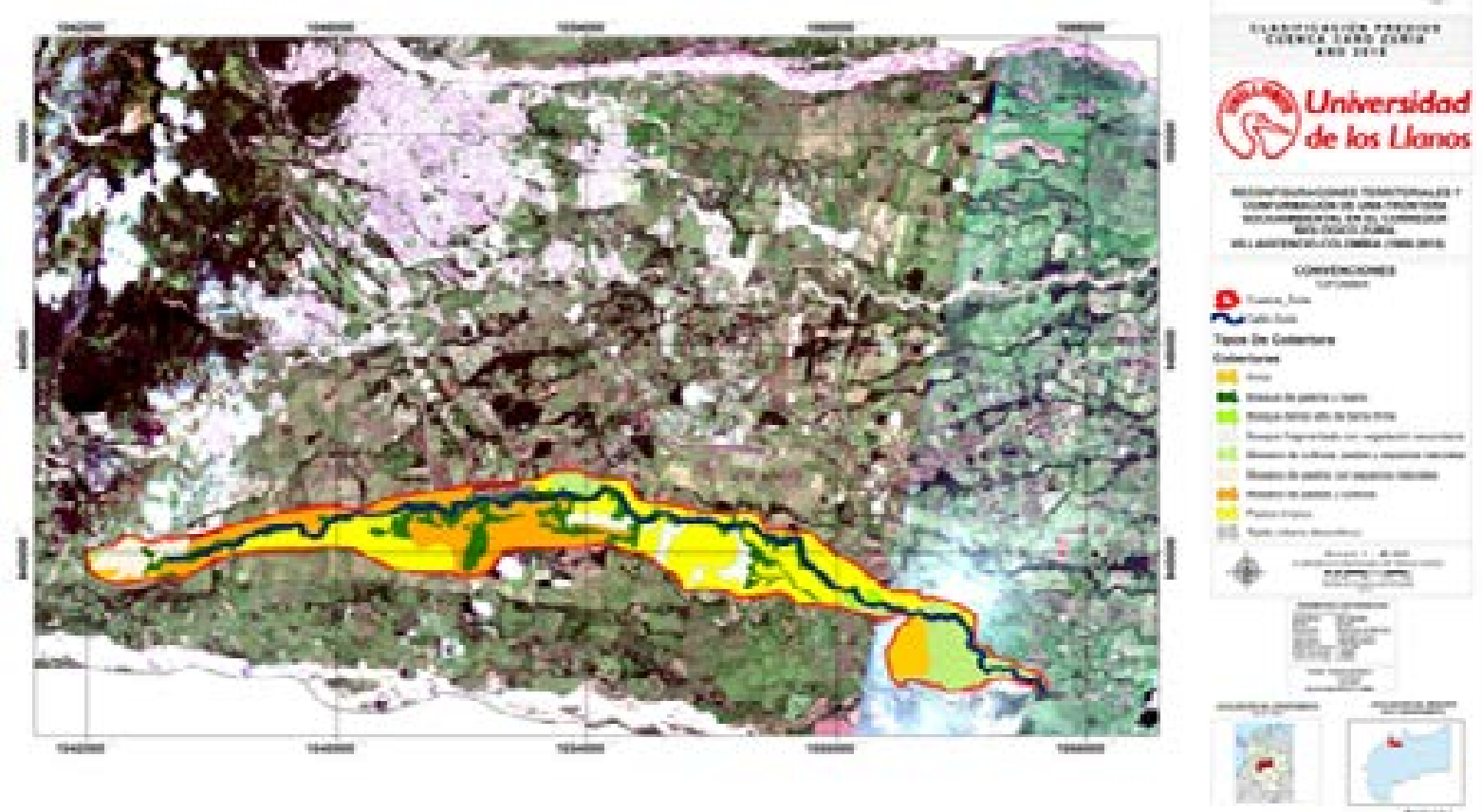

Figura 4. Distribución de coberturas en el área de estudio a 2012. Adaptado de "Estudio de Coberturas Multitemporales 1992-2012", Geodatabase- 2019 por el Instituto Amazónico de Investigaciones Científicas. Fuente: Elaboración Propia. Grupo de Investigación Territorio y Ambiente

En 2012 de acuerdo con la figura 4, se evidencia el sostenimiento en la producción de arroz al mantenerse la extensión de los cultivos de manera aproximada con una variación mínima del $1 \%$ en relación con el periodo anterior, sucediendo lo mismo con la actividad ganadera con una variación del $0,1 \%$ en comparación con el año 2007, lo que deja entrever el sostenimiento en la destrucción del bosque y disminución de la capacidad resiliente en el ecosistema.

La zonificación de Zuria permite conocer el comportamiento espacial de manera más detallada, entender sus dinámicas y hacer un manejo geográfico más extenso y adecuado en cuanto a la conformación socioeconómica, siendo el territorio un espacio que contiene procesos de organización social en los cuales el argumento ambiental está presente para la formación de identidad en ese espacio geográfico (Flórez, 2007).

El resultado de la superposición permitió observar el cambio de dispersión territorial y la perdida de coberturas vegetales con respecto a la expansión del tejido urbano y las actividades agrícolas en la transición de tiempo establecida.

De esta manera se realizó el análisis en dos espacios de tiempo, considerando en un primer escenario el periodo comprendido entre 1992 y 2002 y en el segundo espacio el curso de 2007 a 2015 como se ve a continuación:

De manera dispersa y discontinua es como se ha ido configurando el panorama poblacional, donde no solo la llegada de nuevos moradores sino las necesidades crecientes en la demanda del sector construcción, devienen transformaciones sociales y ambientales con impactos negativos sobre el territorio, comprometiendo la sostenibilidad ambiental de este cinturón verde, lo que nuevamente muestra los datos. 
Tabla 1.

Porcentaje de cambios de cobertura en la cuenca del caño Zuria en la temporalidad de 1992-2012.

\begin{tabular}{|l|c|c|c|c|}
\hline Tipo de Cobertura & 1992 & 2002 & 2007 & 2012 \\
\hline Arroz & $13 \%$ & $16 \%$ & $6 \%$ & $5 \%$ \\
\hline Bosque de galería y ripario & $19 \%$ & $18 \%$ & $17 \%$ & $16 \%$ \\
\hline Bosque denso alto de tierra firme & $10 \%$ & $4 \%$ & $3 \%$ & $2 \%$ \\
\hline $\begin{array}{l}\text { Mosaico de cultivos, pastos y espacios } \\
\text { naturales }\end{array}$ & $38 \%$ & $21 \%$ & $34,9 \%$ & $35 \%$ \\
\hline Pastos enmalezados & $5 \%$ & $4 \%$ & $2 \%$ & $1 \%$ \\
\hline Pastos limpios & $14,8 \%$ & $36,3 \%$ & $36 \%$ & $39,2 \%$ \\
\hline Tejido Urbano & $0,2 \%$ & $0,7 \%$ & $1,1 \%$ & $1,8 \%$ \\
\hline
\end{tabular}

Fuente. Grupo de investigación Territorio y Ambiente 2019

La Tabla 1 contrasta los resultados obtenidos tras el análisis de las figuras 1, 2, 3 y 4, acerca de la zonificación de las coberturas ambientales, junto a las dinámicas territoriales de la cuenca del caño Zuria del municipio de Villavicencio. Contrario a ello, ésta permite evidenciar la disminución en el cultivo de arroz para el año 2012 (ver figura 4), aumentando luego considerablemente $y$ expandiéndose ampliamente en el territorio. Se identificó que el bosque natural en la transición de la temporalidad establecida (1992 a 2015) se encuentra como la cobertura de mayor impacto con respecto a la pérdida, lo que supone un impacto ambiental que genera detrimento en la biodiversidad al disminuir la fertilidad del suelo más la reducción de la cantidad y calidad del agua.

Los bosques denso alto de tierra firme contaban con 3383,08 hectáreas $(\mathrm{Ha}$ ) para el año 1992, las cuales terminaron en $1022 \mathrm{Ha}$ de bosque para el año 2012, mostrando claramente la ampliación de la frontera agrícola por la reforma política del campo en 1992, que buscaba incentivos fiscales y comerciales a los campesinos que se inclinaran en cultivos como el arroz, caso específico para la microcuenca del caño Zuria evidenciado mediante el estudio de zonificación y aplicación de criterios para el uso del suelo establecidos en el Plan de Ordenamiento Territorial (POT), que reconoce del área de mayor afectación.

\section{CONCLUSIONES}

La cobertura vegetal para el año 2015 evidencia un aumento en los mosaicos del cultivo en el corredor biológico el Zuria, donde a pensar que se vio una disminución en su producción de 1992 a 2002, hacia el 2015 este porcentaje de cultivos empieza a incrementar deteriorando el suelo, lo que redunda en erosión, pérdida de nutrientes, deforestación, así como daños en el recurso hídrico en la microcuenca Zuria.

Al evidenciarse que en esta cuenca no se respetan las áreas de retiro a la fuente hídrica, normativa que está establecida en el POT en 300 metros, podría verse afectado el espacio en la que se da una cobertura consistente en mosaico de cultivos, pastos y espacios naturales, bosques fragmentados con vegetación secundaria, cultivos de arroz y pastos limpios, evidenciando una intervención humana que vulnera el ecosistema.

Como resultado de estos procesos de inadecuados usos del suelo, se presenta una fragmentación de la cobertura vegetal pronunciada hacia el año 2000 , aspecto que se 
ha venido acentuando con la tala sistemática de árboles, dejando el territorio con pérdidas en vegetación natural, aunada al creciente aumento del tejido urbano.

Los procesos de dispersión urbana crecientes en el territorio han originado una ocupación extensiva del suelo, lo que ha disminuido los recursos naturales y económicos, al ir por encima de las necesidades propias con el crecimiento de la población que reside en esta zona.

Un territorio transformado a partir de las necesidades que se dan en cada momento histórico ocasiona que las zonas boscosas sean despojadas cada vez que se requiere ampliar la frontera agrícola o pecuaria. Por esto se observa una predominancia de cobertura consistente en mosaicos de pastos y cultivos.

\section{REFERENTES BIBLIOGRÁFICOS}

Álvarez-Icaza Longoria, P. (2014). El uso y la conservación de la biodiversidad en propiedades colectivas: Una propuesta de tipología sobre los niveles de gobernanza. Revista mexicana de sociología, 76(SPE), 199-226.

Arango Marín, M. (2005). Influencia del discurso del desarrollo y las misiones estadounidenses en la formación agronómica (Doctoral dissertation, Universidad Nacional de ColombiaSede Medellín).

Benavides, C. V. (13 de noviembre de 2017). Academia. Obtenido de Academia: https://www.academia.edu/37775115/ Ensayo_sobre_la_Revoluci\%C3\%B3n_ Verde.docx

Bennett, A. F. (2004). Enlazando el paisaje: el papel de los corredores y la conectitividad en la conservación de la vida silvestre. IUCN.
Benayas del Álamo, J. (1992). Paisaje y Educación Ambiental. Evaluación de cambios de actitudes hacia el entorno.

Ceccon, E. (2008). La revolución verde: tragedia en dos actos. Ciencias, 91(091).

Centro virtual de noticias. (2019). Mineducación. Obtenido de mineducación: https:// www.mineducacion.gov.co/cvn/1665/ article-117211.html

FAO. (1996). Enseñanzas de la revolución verde: Hacia una revolución verde. Roma, Italia.

Flórez, M. (2007). La identidad cultural del territorio como base de una estrategia de desarrollo sostenible. Revista ópera, (7), 35-54.

IDEAM, IGAC, CORMAGDALENA. (2007). Mapa de Cobertura de la Tierra Cuenca Magdalena-Cauca, Metodología Corine Land Cover Adaptada para Colombia, escala 1:100.000. Obtenido de IDEAM: http://www.ideam.gov.co/web/ ecosistemas/metodologia-corine-landcover

IDEAM. (2010). Modelogeneral de la metodología Corine Land Cover. Bogotá.

Lefebvre, H., Lorea, I. M., \& Gutiérrez, E. M. M. (2013). La producción del espacio (pp. 31-50). Madrid: Capitán Swing.

Montano, J. (2005). Lifeder. Obtenido de lifeder: https://www.lifeder.com/revolucionverde/

Ochoa, J. (2014). Recuperación de los corredores Biológicos en Villavicencio, caso caño Zuria, valoración económica y social. Villavicencio: Universidad Militar Nueva Granada. 
Santos, M. (2000). La naturaleza del espacio: técnica y tiempo, razón y emoción. Barcelona: Ariel.

Subirós, J. V., Linde, D. V., iPascual,A. L., \& Palom, A. R. (2006). Conceptos y métodos fundamentales en ecología del paisaje (landscape ecology). Una interpretación desde la geografía. Documents d'anàlisi geogràfica, (48), 151-166.

Universidad de Cantabria. (20 de Julio de 2017). Open Course Ware. Obtenido de https://ocw.unican.es/mod/page/view. php?id=979

Universidad Nacional de Colombia. (2016). UNAL. Obtenido de http://ciencias.bogota.unal. edu.co/gruposdeinvestigacion/ecolmod/ informacion-de-interes/que-es-ecologiadel-paisaje/ 